\title{
AOR
}

Selected Papers of \#AoIR2020:

The $21^{\text {st }}$ Annual Conference of the

Association of Internet Researchers

Virtual Event / 27-31 October 2020

\section{Digital Cultures of Care, Safety and Wellbeing}

Anthony McCosker (Chair)

Swinburne University of Technology

Kath Albury

Swinburne University of Technology

Alexia Maddox

Deakin University

Monica Barrett

RMIT University

Christopher Dietzel

McGill University

Practices of self-care and social support have long been identified across different social platforms, as people find new ways of using and adapting digital technologies to mediate and address personal and public health issues. But digital health participation is considerably contested and unevenly experienced, whether through the commodified 'platformization' of the health sector (van Dijck et al., 2018: 98), or in the potentially 'unhealthy' engagement with dominant social media platforms or dating and hookup apps. Contemporary policy frameworks for participatory, digital-enabled healthcare (e.g., NHS, 2019: Ch 5) assume that we all engage in health or help seeking practices online, but have no answers to associated risks of over-exposure, invasive health surveillance or experiences of discrimination and harassment online (Erikainen et al., 2019), particularly for those at the margins. In our case studies, this is pertinent for transgender, non-binary and female hookup app users, people seeking support for

Suggested Citation (APA): McCosker, A., Albury, K., Maddox, A., Barrett, M., Dietzel, C. (2020, October). Digital Cultures of Care, Safety and Wellbeing. Panel presented at AolR 2020: The 21 th Annual Conference of the Association of Internet Researchers. Virtual Event: AolR. Retrieved from http://spir.aoir.org. 
mental ill-health, illicit drug users participating in crypto-markets and dark web communities.

In response to this scenario, this panel asks: what are the forms and capacities for collective care in the current digital ecosystem, between social media platforms and dating apps struggling to address harassment or mental wellbeing, within health service-supported online forums, and across the dark web? This panel looks at evidence and answers, exploring different forms of collective and personal attempts to negotiate, manage, circumvent and otherwise find ways to reinvent cultures of care through digital platforms.

'Cultures of care' is a term coined by Michael Hurley in the early 2000s to account for the collective and relational aspects of dealing with public health crisis. He was interrogating gay men's collective or social practices of 'doing gay' and 'doing sex' within the context of the Australian HIV/AIDS response (Hurley 2003 p.19). For Hurley, 'cultures of care' emerged from networked interconnections between gay men's overlapping practices of love, sex, friendship and intimacy; their relationships to biomedical technologies and systems of knowledge; their interactions with researchers, health policy frameworks, medical professionals and community educators; and their engagement with both 'mainstream' and community/niche broadcast and online media content relating to HIV, gay culture and sexualities. However, Hurley was writing in a time when 'community' was more closely associated with geographical spaces, and social media was still nascent. If cultures of care are based on systems of shared values, how do they manifest within digital culture? Across platforms? In relation to the fragmentation of identity categories? Between online communities of practice and researchers?

Picking up Hurley's invitation to consider the "practices and repertoires of everyday life" as key aspects of cultures of care, this panel investigates the relationships between digital media platforms and practices, health policy, health promotion, research ethics and knowledge co-creation - as these come to affect the kinds of connection and community that matters for care and wellbeing in the digital age.

\section{References}

Erikainen, S., Pickersgill, M., Cunningham-Burley, S., \& Chan, S. (2019). Patienthood and participation in the digital era. Digital Health, 5, 2055207619845546.

Hurley, M. (2003) Then and Now. Gay Men and HIV. Monograph Series Number 46. The Australian Research Centre in Sex, Health \& Society, La Trobe University, Melbourne, Australia 
Hurley, M. (ed) (2002) Cultures of Care And Safe Sex Amongst HIV Positive Australians. Papers from the HIV Futures I and II surveys and interviews, Monograph Series No. 43, Australian Research Centre in Sex, Health and Society, La Trobe University, Melbourne.

NHS (2019) The NHS Long Term Plan, https://www.longtermplan.nhs.uk/.

Van Dijck, J., Poell, T., \& De Waal, M. (2018). The platform society: Public values in a connective world. Oxford University Press. 


\section{Going off site and off script: Social support and health participation at the digital margins}

Alexia Maddox

Deakin University

Anthony McCosker

Swinburne University of Technology

Monica J. Barratt

RMIT University \& UNSW Sydney

Online communities offer new forms of health participation, community-based support at scale (McCosker, 2018) and the opportunity for targeted health-messaging (White, 2001) and intervention. Health empowerment plays out in specific online contexts; for people experiencing mental health challenges, one such space is the beyond blue forums (McCosker, 2018). People seeking a safe space for anonymous discussion of drug use, can access online drug discussion spaces in the clear net (Barratt, 2011) or cryptomarket forums in the dark web (Maddox et al., 2016). These platforms support transparent exchanges around mental health, wellbeing and drug use that may be stigmatised and unacceptable in other more mainstream online or in person interactions. While research has established the value of online social support (Shaw, 2002; Kraut, 2002) and safe spaces online for vulnerable populations (Hanckel and Morris, 2014; Wilson, 2017), the curation and monitoring practices within digital platforms can be counter-productive.

\section{Methods and Approach}

This paper presents evidence from two empirical studies of the Beyond Blue mental health support forum and participants from the Silk Road cryptomarket forums. Participants of these forums recount their movement toward the digital margins ('offsite'), and away from traditional health institutions and services, in order to go 'off script'. The empirical basis for this work is founded on the use of digital ethnography in the two case study populations, combining observations over time with participant interviews.

The first case examines activity across Beyond Blue's online support sites over several years (2017-2018). The starting point was to identify and gain access to key informants and prominent, influential forum participants, and then observe practices, events and activities in the forums over time. Interviews were conducted with 10 participants. The second case study draws from collaborative research undertaken between 2013 and 2014 exploring the social implications of cryptomarkets, focusing on the leading cryptomarket, Silk Road. The Silk Road study incorporated multi-sited observation and engagement with the community surrounding Silk Road, including 17 interviews. It 
focuses on an anonymous population of drug users and adds the capacity for

cryptomarket-based action with alternative modes of governance and autonomy.

These two cases point to different but interrelated sites of collective attempts to manage imposed, scripted forms of health care, in favour of developing a culture of collective care that is always pushing against medical frames and platform structures.

\section{Findings and discussion}

In both cases, participants sought forms of radical transparency in their practices, whilst protecting their identities, and moved to decentralised contexts in order to enact selfauthored versions of collective care outside of surveilled channels.

The Beyond Blue forum enables access, with anonymity under increasing pressure for platforms to moderate, force real-name participation, and share data with authorities (to prevent suicide for example). Explaining her involvement with the forum, Just Sara emphasises the social pressures of discussing her mental ill-health on Facebook:

I did make one little comment to say that I haven't been well, not dealing with things too well [...] My Aunty wrote back and blurted this big spiel about having a mental illness isn't a bad thing, and just went on and on ... and I was so put back by it, I haven't posted since. (Just Sara, Beyond Blue forum)

We argue that these kinds of practices are more than about managing privacy. They are also finding or managing digital environments that enable cultures of care. Platform surveillance, the curation and control of the conversational 'scripts' and the dominant values and codes imposed by both health institutions or clinical practice, and social media and online health platforms, can have the reverse effect of excluding vulnerable populations who wish to go 'off script'.

The exit movement documented across these two cases indicates a spectrum of action from information-sharing and social support to resource sharing and active selfdiagnosis and treatment. Through this empirical consideration we argue there is an unresolved tension or contradiction in the high-level demand for participatory and community health technology that both our cases insistently pinpoint. To illustrate this contradiction, we identify a range of factors related to people's fraught relationships with face-to-face (F2F) healthcare and treatment forms, and with information sources and interfaces.

In one example from a Silk Road participant, the lines between sanctioned pharmaceuticals and medicinal drugs restricts attempts to self-manage their health: 
I'm prescribed some ADD [Attention Deficit Disorder] meds, but my doctor won't prescribe me the ADD meds that I know work much better for me, because it has a high street value and abuse potential. So I just bought it through the Silk Road

I think with things like marijuana and psychedelics a huge amount of people were able to access those substances not just to get high, but either for medical use or just for exploring themselves. (Participant 19, Silk Road)

The off site, off script practices considered highlight the agency of online participants to utilise the affordances of the environment to construct a more decentralised and permissive community of care based upon radical transparency and emerging technologies.

\section{References}

Barratt, M.J. (2011) Discussing illicit drugs in public internet forums: Visibility, stigma, and pseudonymity, in J Kjeldskov \& J Paay (eds), C\&T'11. Proceedings of the Fifth International Conference on Communities and Technologies, Brisbane, Australia, ACM, New York, NY, pp. 159-68

Hanckel, B. \& Morris, A. (2014) Finding community and contesting heteronormativity: queer young people's engagement in an Australian online community, Journal of Youth Studies, vol. 17, no. 7, pp. 872-86.

Kraut, R., Kiesler, S., Boneva, B., Cummings, J., Helgeson, V. \& Crawford, A. (2002) 'Internet Paradox Revisited', Journal of Social Issues, vol. 58, no. 1, pp. 49-74.

Maddox, A., Barratt, M.J., Lenton, S. \& Allen, M. (2016) Constructive activism in the dark web: cryptomarkets and illicit drugs in the digital 'demimonde', Information Communication and Society, vol. 19, no. 1, pp. 111-26.

McCosker, A. (2018) Engaging mental health online: Insights from beyondblue's forum influencers, New Media \& Society, vol. 20, no. 12, pp. 4748-64.

Shaw, L.H. \& Gant, L.M. (2002) In defense of the internet: the relationship between Internet communication and depression, loneliness, self-esteem, and perceived social support, Cyberpsychology \& Behavior: The Impact Of The Internet, Multimedia And Virtual Reality On Behavior And Society, vol. 5, no. 2, pp. 157-71.

White, M. \& Dorman, S.M. (2001) Receiving social support online: implications for health education, Health Education Research, vol. 16, no. 6, pp. 693-707.

Wilson, A., Carlson, B.L. \& Sciascia, A. (2017), 'Reterritorialising Social Media: Indigenous People Rise Up', Australasian Journal of Information Systems, vol. 21, no. 0. 


\section{Disconnective practices as situated cultures of care and wellbeing in dating app use}

Kath Albury

Swinburne University of Technology

Anthony McCosker

Swinburne University of Technology

Daniel Reeders

Australian National University

Dating and hookup apps like Tinder, Grindr and Bumble are deeply implicated in the transformation of intimacy and the mobile mediation of relationships (e.g. Albury \& Byron, 2016; Duguay, 2017; Hobbs, Owen \& Gerber, 2017; Møller \& Petersen, 2018). But users' relationship with and through apps are far from seamless and untroubled.

While they have become ordinary, everyday technologies of mediated relationships, dating apps have raised significant issues regarding health and wellbeing, including risks of sexual assault, harassment and sexually transmitted infections (STIs), along with personal data and privacy breaches (McCosker et al., 2019). In fact, their status as 'technologies of risk' (Albury \& Byron, 2016) has only risen over the past decade as population health research has sought to pin rising STIs to app use (e.g., Rice et al., 2012; Sawyer et al., 2018). Similarly, a number of initiatives have been put in place by app producers to reduce harassment, improve safety, and support sexual health messaging (for example Grindr's 'Kindr' campaign addressing racism and sexism and discrimination on the app). It is not clear, however, that these issues can be addressed by platforms directly through campaigns or technological interventions alone.

There is a need for critical interrogation of wellbeing and safety in app use that incorporates the voices and experiences of app users, and tests mediating factors such as gender, sexuality, mental health alongside cultures of use.

\section{Methods}

To address this gap, this paper draws on a two-year study of the safety, risk and wellbeing in experiences of mobile dating apps. The study involves a mixed-methods approach that included a survey of app users across all Australian states and territories $(\mathrm{N}=382)$, workshops with 18-35-year-old app users $(\mathrm{N}=51)$, and in-depth interviews $(\mathrm{N}=10)$. We focus on specific quantitative and qualitative findings in this study that show how wellbeing and safety are not evenly experienced by dating and hookup app users. We show that wellbeing is intimately bound up with a set of tensions around the sociotechnical components that shape experiences of connection and disconnection. 


\section{Approach and Findings}

Our approach to digital wellbeing draws on and extends Atkinson's (2013) account of wellbeing as a relational and situated assemblage. Atkinson frames wellbeing as 'an affect, depended on the mobilisation of resources from everyday encounters with complex assemblages of people, things and places' (2013, p. 137). A relational concept of wellbeing is vital, we argue, for understanding and facilitating cultures of care, safety and wellbeing in dating app use.

In the quantitative component, survey participants ( $n=382$, age 15-35) were recruited via unpaid posts on the social networks of the researchers and organizational partners. Participants could select gender and sexual identities or describe their own in open-text. Baseline health and disability were assessed using validated scales - the SF-8 and WG-SS, respectively. Respondents listed the apps they ever, currently, and most used, and answered multi-choice questions on purposes, duration and intensity of use, satisfaction, concerns, experiences of discrimination, harassment, and unsafety, and safe sex practices. Rather than dividing participants into a matrix of gender and sexual identity categories, quantitative analysis drew on the literature and qualitative findings to identify factors that might predict differences in experiences and outcomes of app use, including sex and gender diversity, cultural diversity, disability, and anxiety.

Results identify both needs and potential sites for health promotion intervention. Limitations include very low recruitment of heterosexual men $(n=10)$ and a convenience sample composed of people already connected with health promotion services. Survey data showed that experiences of feeling safe or unsafe when using dating apps vary with socio-cultural experiences of identity, sexuality and gender. Those who are marginalised or vulnerable in other ways (e.g., due to stigma associated with physical and mental ill-health, ableism, ageism, misogyny, homophobia, racism and transphobia) are also likely to feel unsafe when using apps. As we found, some groups use and appreciate apps in different ways, including people living with higher than average anxiety or with disability.

The qualitative findings, similarly, show that dating apps fulfil important objectives for people who experience marginalisation and vulnerability. Some participants discussed taking breaks from app use during times of stress or when focus was needed elsewhere, such as during exams. Turning off notifications was a common self-care strategy:

I turn notifications off when I've got other stuff going on and I make the choice to go in and use it because notifications are big for me. I think a lot of people are the same; if it comes up, it's like oh, you've got to respond straight away, or like have to be using it.' (26, gay, male, urban) 
Temporary deletion of apps was a common response to negative experiences:

So many of my friends regularly will take app breaks. It's also often a question with breakups - 'are you ready to be on the apps yet?' Much like people will try to detox from technology on the whole, people are recognising the negative aspects of dating apps and trying to detox specifically from them. (27, queer, female, urban)

Overall, app use was framed as an 'ordinary' part of contemporary relationships for our participants, which could, in some cases increase their sense of connection, community and pleasure. However, some users experienced frustration, anxiety and distress. In line with previous research (Light, 2014; Light and Cassidy, 2014) we found a range of disconnective practices and that helped participants manage connection through techniques such as careful profile management, taking breaks (deleting and reinstalling apps), linking or keeping other social media accounts separate, negotiating spaces for meeting up, and even engaging in forms of distributed cultures of care and support around app use and relationships.

These findings make sense if we consider Atkinson's account of wellbeing as situated and relational. They signal the importance of personal and collective strategies of support, care and safety as they interact with platform features, technical rules, physical spaces, and sanctions and cultures of app use.

\section{References}

Albury, K., \& Byron, P. (2016) Safe on my phone? Same-sex attracted young people's negotiations of intimacy, visibility, and risk on digital hook-up apps. Social Media+ Society, 2(4), 2056305116672887.

Atkinson, S. (2013) Beyond components of wellbeing: The effects of relational and situated assemblage. Topoi, 32(2), 137-144.

Duguay, S. (2017). Dressing up Tinderella: Interrogating authenticity claims on the mobile dating app Tinder. Information, Communication \& Society, 20(3), 351-367.

Hobbs, M., Owen, S., \& Gerber, L. (2017) Liquid love? Dating apps, sex, relationships and the digital transformation of intimacy. Journal of Sociology, 53(2), 271-284.

Jørgensen, K. M., \& Petersen, M. N. (2018) Bleeding boundaries: Domesticating gay hook-up apps. In Mediated Intimacies: Connectivities, Relationalities and Proximities (pp. 208-223). Routledge.

Light, B. (2014). Disconnecting with social networking sites. Springer, London. 
Light, B., \& Cassidy, E. (2014) Strategies for the suspension and prevention of connection: Rendering disconnection as socioeconomic lubricant with Facebook. New Media \& Society, 16(7), 1169-1184.

McCosker, A., Albury, K., Pym, T., Byron, P., Race, K. (2019) Swiping, stealthing and catfishing: dating and hookup apps in the media research report, Swinburne University of Technology, Melbourne. http://doi.org/10.25916/5d7ed40274311

Rice, E., et al. (2012), Sex risk among young men who have sex with men who use Grindr, a smartphone geosocial networking application, Journal of AIDS \& clinical research, Suppl. 4, pp. 1-8.

Sawyer, A.N., Smith, E.R. \& Benotsch, E.G. (2018) Dating application use and sexual risk behavior among young adults, Sexuality Research and Social Policy, 15(2), 183191. 


\section{Dating Beyond Binaries: Trans and Gender-Diverse Dating App Users Negotiations of Safety, Risk and Wellbeing}

Christopher Dietzel

McGill University

Trans and gender-diverse (TGD) people face high risks of dating violence, both online and in person (Dank et al., 2014; Walls et al., 2019). This paper draws on qualitative interviews and creative workshops conducted with Australian TGD dating app users aged 18-35 $(n=13)$, and consultations with professionals in Australian sexual health organisations and TGD-specific organisations $(n=22)$. It explores how TGD dating app users negotiate their safety, health, and well-being - and, in doing so, aims to offer insights into the specific needs and safety concerns of TGD dating app users.

\section{Background}

Research into TGD dating experiences suggests that at least half of trans people have reported unwanted sexual activity (Stotzer, 2009), and over half have suffered psychological abuse (Turell, 2000). Six in ten have experienced sexual coercion, and nearly nine in ten have faced physical violence (Dank et al., 2014). This is not news to TGD dating app users who have recorded their concerns in blog posts and social media (McKeon, 2015; Windust, 2018) and have outlined strategies they employ to promote safety (Sonoma, 2019). The lessons learned and peer advice shared by these TGD dating app users promotes a culture of care through which members of the community learn from one another in order to minimize risks when using dating apps (Wong, 2018).

\section{Findings}

TGD participants in our study used a range of popular apps, including Tinder, Grindr, HER, and OkCupid. Although participants held diverse understandings of safety, health, and wellbeing and applied different strategies to navigate potential risks, there were common strategies participants employed that helped them feel safer on apps and in person. As in previous studies of app users, participants deployed practices of what Duguay (2017) has termed 'identity modulation' to promote a sense of wellbeing and safety. However, this practice was complicated for those who were dating while in the process of exploring their gender identity or actively transitioning:

I started out using things like Craigslist, Reddit, FetLife. Basically, I was more interested in using things that weren't picture and profile sort of things. I didn't want to show myself. I was like, cool, if I can just put it into words and someone's 
interested in what I have to say, and what I can say about myself - because I was not ready to put my face onto a thing if someone saw me. (Blair, she/her)

I probably had a bit of a break when I was transitioning early on because I didn't have a lot of confidence, and then just didn't feel that I was probably worthy enough, which is a bit stupid but l'm still working on that. (Tristan he/him)

As Bivens. and Haimson (2016) have observed, binary gender has been 'baked in' to the design of many social media platforms - including popular dating and hook up apps. This causes particular difficulties for participants who sought to avoid contact with abusive, transphobic or fetishizing individuals; and also limited users' opportunities to deliberately seek out other TGD app users for the purposes on dating, friendship and community-building. As Blair explained:

Tinder has options to put all the specific gender, and showcase - are you a translesbian and all this stuff. ... The only problem is that you can't siphon it down to other people who you're interested in very well. It's like, yeah, it's just interested in men or women. Those are your two choices for most of those apps.

\section{Discussion and Conclusions}

Participants described a range of strategies for addressing concerns and minimizing perceived risks. For example Max (they/them) described their practice of maximising opportunities for connection with other queer and TGD people on Tinder by logging in as a 'man seeking men' on some occasions and a 'woman seeking women' on others. Several users also described cycling through a range of 'straight' and 'queer' apps as they explored their evolving sexual desires and identities at different points in time, describing their relative drawbacks and merits. Apps such as Feeld and OKCupid that facilitate longer, more nuanced profiles were preferred by users seeking non-dyadic or polyamorous relationships.

While interacting with other dating app users, participants relied on the affordances of apps as well as cues from user profiles, photos, and chats to assess potential risks and 'red flags'. Participants and professional reference group members noted that TGD app users have specific wellbeing concerns that are not always shared within the broader LGBTIQ+ community. For example, Parker (he/him) expressed frustration with friends who encouraged him to take what he considered to be risks when using apps:

My cis friends are like, it'll be fine. I'm like, you don't know. They don't understand. I could get killed. But they're like, just do it. 
To date, few studies have investigated the resources and supports available to TGD people through sexual health organisations, and fewer still have addressed the relationship between dating app use and healthcare/social support. The findings and recommendations from this study provide preliminary insights into the contemporary digital dating cultures of TGD people and outline tangible concerns that sexual health organisations can address in order to promote the safety, health, and well-being of TGD people.

\section{References}

Bivens, R. and Haimson, O.L., (2016). Baking gender into social media design: How platforms shape categories for users and advertisers. Social Media+ Society, 2(4), p.2056305116672486.

Dank, M., Lachman, P., Zweig, J. M., \& Yahner, J. (2014). Dating violence experiences of lesbian, gay, bisexual, and transgender youth. Journal of Youth and Adolescence, 43(5), 846-857.

Duguay, S., (2017). Identity modulation in networked publics: Queer women's participation and representation on Tinder, Instagram, and Vine (Doctoral dissertation, Queensland University of Technology).

McKeon, L. (2015, December 17). What it's really like to be young and trans in Canada now. FLARE. https://www.flare.com/tv-movies/what-its-really-like-to-be-young-andtrans-in-canada-now/

Risser, J. M. H., Shelton, A., McCurdy, S., Atkinson, J., Padgett, P., Useche, B., et al. (2005). Sex, drugs, violence, and HIV status among male-to-female transgender person in Houston, TX. In Walter Bockting \& Eric Avery (Eds.), Transgender health and HIV prevention: Needs assessment studies from transgender communities across the United States. Binghamptom, NY: Haworth Medical Press.

Sonoma, S. (2019, February 14). What dating and love is like for 10 nonbinary people. Teen Vogue. https://www.teenvogue.com/story/what-dating-and-love-is-like-for-10-nonbinary-people

Stotzer, R. L. (2009). Violence against transgender people: A review of United States data. Aggression and Violent Behavior, 14(3), 170-179.

Turrell, S. (2000). A descriptive analysis of same-sex relationship violence for a diverse sample. Journal of Family Violence, 15, 281-293. 
Walls, N. E., Atteberry-Ash, B., Kattari, S. K., Peitzmeier, S., Kattari, L., \& LangenderferMagruder, L. (2019). Gender identity, sexual orientation, mental health, and bullying as predictors of partner violence in a representative sample of youth. Journal of Adolescent Health, 64(1), 86-92.

Windlust, J. (2018, July 17). 5 lessons you learn when you date as a non-binary person. Cosmopolitan. https://www.cosmopolitan.com/uk/love-sex/relationships/a22211856/nonbinary-dating/ 


\title{
A Middle Ground Between Fair Game and Locked Down: Bluelight.org, Illegal Drug Use, and Digital Media Research Ethics
}

\author{
Monica Barratt \\ RMIT University and University of New South Wales \\ Michael Gilbert \\ Independent

\section{Background}

The question of how to evaluate and ensure ethical practices in social media research continues to be much debated. For some, publicly accessible digital traces of human interactions are considered as public documents comprising public information, and therefore outside the remit of human subjects research (see Zimmer, 2010). For example, the policy governing research ethics in the US, The Common Rule, uses a decision tree to determine whether an activity is 'research involving human subjects', and the (passive) analysis of publicly accessible digital data that does not contain personally identifying information is deemed to fall outside this category (see Vayena, Gasser, Wood, O'Brien, \& Altman, 2016). In contrast, others (Chiauzzi \& Wicks, 2019) have demonstrated that humans producing these digital traces can be affected and harmed by inclusion in research that analyses personally identifiable information without their knowledge, thereby complicating the exemption from ethical review and compliance under the Common Rule.

Such decisions often rest on determining whether or not data are public or private, without acknowledgement of the complexities of making this determination.

Nissembaum's concept of 'contextual integrity' (Nissenbaum, 2010) troubles the seeming public-private dichotomy. People who provide data on digital platforms have an interest in "ensuring it flows appropriately" (Nissenbaum, 2010). What is technically considered publicly available data may be sensitive, personal or both, and its capture and use may prompt additional ethical dilemmas (Ravn, Barnwell, \& Barbosa Neves, 2020). Agreeing to the re-use of one's data for research purposes in a terms of use agreement may be inadequate as a method of informed consent (Halavais, 2019; Vayena, Mastroianni, \& Kahn, 2013), particularly as the scope, focus, and products of research evolve over time. Changes in analytical methods and focus over time may also impact the extent to which anonymous or pseudonymous data may be deanonymized, with differences in data structuring analysis leading to critical implications for applicability of the Common Rule's definition of 'identifiable private information'.

Another factor that requires consideration, in addition to the researcher and the individual participants, is the impact on communities or platforms within which the digital traces occur (Chiauzzi \& Wicks, 2019). The community itself has a key interest in 
whether research is conducted, how it is represented, and in the protection of its members' privacy and sustained interactions. In some cases, there is a fourth actor involved: the platform which hosts the community, e.g. Facebook or Twitter (companies that have their own commercial interests and whose actions have stifled critical research; Bruns, 2019). Twitter has recently tightened its rules about what researchers can do with its data (see Twitter, 2020). It is clear that we must also consider the interests of communities and corporate entities that develop and operate online platforms as we evaluate individual cases of data use for research purposes.

Vayena and Tasioulas (2016) provide a useful framework for balancing two human rights that relate to this question: the right to privacy and the lesser-known right to science. In their analysis of human rights declarations, their reading of the right to science is not only that humans have a right to benefit from scientific advances, but also that 'people have a right to actively participate in scientific inquiry' (Vayena \& Tasioulas, 2016, p. 4). Indeed, the emergence of networked digital technologies has facilitated what has been called 'citizen science', including citizen-led research (Vayena \& Tasioulas, 2015).

If social media datasets are not necessarily fair game for research activities, how should the ethical practice of research be evaluated and ensured? Can the passive contribution of data to a research project without informed consent of research aims or outputs be considered full participation? What if obtaining informed consent or ensuring beneficence for thousands (or millions) of individuals is simply impractical?

\section{The Bluelight case study}

Bluelight administrators describe the site as "an international, online, harm-reduction community committed to reducing the harms associated with drug use". The site's longevity is exceptional, having just celebrated its 20-year birthday. The site has hosted discussion forums since inception and keeps a large archive of drug-related discussion content spanning this entire period. Bluelight's structure as a stand-alone website means that there is no commercial platform that requires additional consideration. In this paper we draw on our experiences managing research partnerships on behalf of Bluelight and liaising with data customers with an interest in its dataset. In the discussion, we draw out learnings from this case that will be of wider benefit when considering the ethics of digital research with stigmatised populations and on sensitive topics more generally.

Many online communities may reject offers to partner with researchers or may simply not have been asked. Bluelight has supported research because it provides a win-win through enabling open dialogue between researchers and consumers. Historically, many researchers will 'do it anyway' if the community doesn't actively create a pathway for engagement. The 'do it anyway' examples can be found in publications in academic 
journals where Bluelight's contribution has been erased in the spirit of protection: both to protect privacy of users and to help allow researchers to continue non-consented research, but the community has been able to identify itself (thus the 'protection' was inadequate, as well as being unwelcomed by the community). Importantly, research is Bluelight's primary source of revenue. Without research Bluelight would not be able to pay its server costs and would not be able to keep the website free of paid advertising. Additionally, the community wants to support research into drug-related harm reduction that might both challenge stereotypes and improve the health of people who use drugs. To that aim, Bluelight requires researchers to participate in collaborative oversight of research practices.

When evaluating a research topic, Bluelight will deny authorisation to research deemed mis-aligned with its mission. Due to the infrastructural burdens of web scraping and the lack of control over the data that is scraped, Bluelight prefers to provide researchers directly with a tailored data file. Funded or commercial research partners are asked to contribute an appropriate donation to help fund the website, while students and unfunded researchers may also donate small amounts. Bluelight advises researchers who wish to present verbatim quotations to contact contributors directly and present the quotation in context, then offer them options for attribution or anonymisation. In the case of non-consent, quotes may be altered enough so as not to lead back to the original quote via search engine (Wilkinson \& Thelwall, 2011) or used in a composite fashion to create an exemplar (see Markham, 2012). Bluelight also offers researchers a more involved partnership opportunity, where Bluelight can offer collaboration on research grants, significant contribution to research outputs (co-authorship), opportunities to get regular feedback on emerging findings from the private moderators sub-forum, or to create a new private sub-forum specifically for the research project where the researcher can interact with a selected group of community members.

\section{Discussion}

In the case study of Bluelight, the community administrators play a pivotal role in negotiating the ethical parameters of research projects. It is a stark example of how frameworks that focus solely on how researchers interact with individual participants or contributors to a website/community may fall short in meeting community expectations. Vayena et al.'s (2016) interpretation of the human right to science being one that includes a right to participate in research prompts serious consideration by ethics committees and researchers in this space. As social media platforms and third-party vendors develop an array of data use agreements and protections, the case study of Bluelight illustrates one pathway towards striking a balance between fair game and 
locked down which could be drawn upon by other researchers and digital communities where topics are sensitive and/or identities are stigmatised.

\section{References}

Bruns, A. (2019). After the 'APIcalypse': social media platforms and their fight against critical scholarly research. Information, Communication \& Society, 22(11), 1544-1566.

Chiauzzi, E., \& Wicks, P. (2019). Digital trespass: ethical and terms-of-use violations by researchers accessing data from an online patient community. Journal of Medical Internet Research, 21(2), e11985.

Halavais, A. (2019). Overcoming terms of service: a proposal for ethical distributed research. Information, Communication \& Society, 22(11), 1567-1581.

Markham, A. (2012). Fabrication as ethical practice. Qualitative inquiry in ambiguous internet contexts. Information, Communication \& Society, 15(3), 334-353.

Nissenbaum, H. (2010). Privacy in context. Technology, policy, and the integrity of social life. Stanford: Stanford Law Books.

Ravn, S., Barnwell, A., \& Barbosa Neves, B. (2020). What Is "publicly available data"? Exploring blurred public-private boundaries and ethical practices through a case study on Instagram. Journal of Empirical Research on Human Research Ethics, 15, 40-45.

Twitter. (2020). More about restricted uses of the Twitter APIs. Retrieved from https://developer.twitter.com/en/developer-terms/more-on-restricted-use-cases

Vayena, E., Gasser, U., Wood, A., O'Brien, D., \& Altman, M. (2016). Elements of a new ethical framework for big data research. Washington and Lee Law Review Online, 72(3), Article 5.

Vayena, E., Mastroianni, A., \& Kahn, J. (2013). Caught in the web: informed consent for online health research. Science Translational Medicine, 5(173), 173-176.

Vayena, E., \& Tasioulas, J. (2015). "We the scientists": a human right to citizen science. Philosophy \& Technology, 28(3), 479-485.

Wilkinson, D., \& Thelwall, M. (2011). Researching personal information on the public web: Methods and ethics. Social Science Computer Review, 29(4), 387-401.

Zimmer, M. (2010). "But the data is already public": On the ethics of research in Facebook. Ethics \& Information Technology, 12, 313-325. 
